\title{
EXTENSIONS OF GROUP RETRACTIONS
}

\author{
RICHARD D. BYRD, JUSTIN T. LLOYD \\ ROBERTO A. MENA, AND J. ROGER TELLER
}

\author{
Department of Mathematics \\ University of Houston \\ Houston, Texas 77004 U.S.A.
}

(Received June 18, 1979 and in revised form February 29, 1980)

ABSTRACT. In this paper a condition, which is necessary and sufficient, is determined when a retraction of a subgroup $\mathrm{H}$ of a torsion-free group $\mathrm{G}$ can be extended to a retraction of $G$. It is also shown that each retraction of a torsion-free abelian group can be uniquely extended to a retraction of its divisible closure.

KEY WORDS AND PHRASES. Group retraction, extending group retractions, torsionfree abelian group, divisible closure. .

1980 MATHEMATICS SUBJECT CLASSIFICATION CODES. Primary, 06A75, 20 E99 Secondary, 20K15, 20K20.

\section{INTRODUCTION.}

The concept of a retractable group was introduced in [2] and there it was shown that the class of lattice-ordered groups is a proper subclass of the class of retractable groups. It was shown in [4] that group retractions induce "nonstandard" automorphisms of the semigroup of finite complexes of a torsion-free 
abelian group. In this paper we consider the following question: if $G$ is a torison-free abelian group, $H$ is a subgroup of $G$, and $\tau$ is a retraction of $H$, then when can $\tau$ be extended to a retraction of $G$ ? In Theorem 5.7 we give a necessary and sufficient condition for the existence of such an extension. The key to proving this theorem is Theorem 3.2 where it is shown that if $H$ is a normal subgroup of a group $G$ such that $G / H$ can be linearly ordered and $\tau$ is a retraction of $H$ such that for each $g \in G$ and each finite nonempty subset $A$ of $H,\left(g^{-1} A g\right) \tau=g^{-1}(A \tau) g$, then $\tau$ can be extended to a retraction of G. This theorem is a generalization of a corresponding theorem in the theory of lattice-ordered groups (see $[1],[7]$, or [10]).

If $G$ is a torsion-free abelian group and $D$ is a divisible closure of $G$, then, in Theorem 4.7, we show that each retraction of $G$ has a unique extension to a retraction of $D$. Again this theorem generalizes a well-known result in the theory of lattice-ordered groups. The key in the proof of Theorem 4.7 is Corollary 4.4 where it is shown that each retraction of an abelian group satisfies condition $(\delta)$. (Definitions will be given in Section 2.) In Corollary 4.8, we obtain a partial converse of Theorem 4.7. An immediate consequence of Corollary 5.8 is that each torsion-free abelian group admits an infinite number or retractions.

\section{PRELIMINARIES.}

In this section we give some definitions and results from [2] that will be used in this paper. Throughout this paper, $G$ will denote a group,written multiplicatively and with identity $l$, and F(G) will denote the collection of all finite, nonempty subsets of $G$. Then $F(G)$ is a join monoid, that is, $F(G)$ is a join semilattice in which $A \vee B=A \cup B, F(G)$ is a monoid in which $A B=\{a b \mid a \in A$ and $b \in B\}, A(B \vee C)=A B \vee A C$, and $(A \vee B) C=A C \vee B C . A$ homomorphism $\sigma$ of $F(G)$ into $G$ such that $\{g\} \sigma=g$ for every $g \in G$ will be called a retraction of $G$. We will denote by Ret $G$ the collection of all 
retractions of $G$. If Ret $G$ is nonempty, then $G$ is said to be a retractable group. The class of retractable groups is a proper subclass of the class of torsion-free groups [2, Theorem 2.2 and Example 2.7].

If $G$ is a lattice-ordered group and $\sigma$ is given by $A \sigma=V$ for each $A \in F(G)$, then $\sigma \in \operatorname{Ret} G[2$, Theorem 2.1] and $\sigma$ is called the retraction of $G$ induced by the lattice-ordering of $G$.

Let $\sigma \in \operatorname{Ret} G$. Then the kernel of $\sigma$, denoted by Ker $\sigma$, is the set $\{A \mid A \in F(G)$ and $A \sigma=\imath\}$. If Ker $\sigma$ is a convex subset (resp., convex subsemilattice) of $F(G)$, then $\sigma$ is said to be a convex retraction (resp., $\ell-\underline{r e-}$ traction) of $\mathrm{G}$. There is a one-to-one correspondence between the latticeorderings of $G$ and the l-retractions of $G$ [2, Corollary 3.3]. In [2, Theorem 3.2] six conditions are given, each of which is equivalent to $\sigma$ being an $\ell-r e-$ traction. A subgroup $H$ of $G$ is said to be a $\sigma$-subgroup if $A \sigma \epsilon$ for every $A \in F(H)$. In this paper retractions that satisfy the following condition are of prime importance:

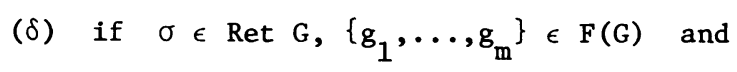

$\mathbf{n}$ is a natural number, then

$$
\left\{g_{1}^{n}, \ldots, g_{m}^{n}\right\} \sigma=\left(\left\{g_{1}, \ldots, g_{m}\right\} \sigma\right)^{n}
$$

If $G$ is a lattice-ordered group and $\sigma$ is the retraction induced by the latticeordering of $G$, then $\sigma$ satisfies $(\delta)$ if and only if the lattice-ordering is representable. (This can be proven by [5, Theorem 1.8].) In [3] examples were given of groups that admit retractions satisfying $(\delta)$, but do not admit latticeorderings.

Let $\sigma \in \operatorname{Ret} G$ and $H$ be a subgroup of $G$. Then $H$ is said to be a $\rho-\sigma$-subgroup of $G$ if $A=\left\{g_{1}, \ldots, g_{n}\right\} \in F(G)$ and $h_{1}, \ldots, h_{n} \in H$ implies that $H(A \sigma)=H\left(\left\{h_{1} g_{1}, \ldots, h_{n} g_{n}\right\} \sigma\right)$. Every $\rho-\sigma$-subgroup of $G$ is pure $[2$, Corollary 4.10] and is a o-subgroup [2, Theorem 4.2].

The natural numbers will be denoted by $N$, the integers by $Z$, and the 
rationals by $Q$. If $A \subseteq G$ and $n \in N$, then

$$
A^{n}=\left\{a_{1} \ldots a_{n} \mid a_{1}, \ldots, a_{n} \in A\right\}
$$

and

$$
A^{(n)}=\left\{a^{n} \mid a \in A\right\} .
$$

3. LEXICOGRAPHIC EXTENSIONS.

In 1942, F. W. Levi [10, p. 260] (or see $[1$, p. 289] or $[7$, p. 20]) gave a necessary and sufficient condition that a partial order of a subgroup of a group may be extended to a partial order of the group. The main result of this section gives a sufficient condition for the extension of a retraction of a subgroup to a retraction of the group. The proof of Theorem 3.1 is immediate and will be omitted.

THEOREM 3.1: Let $\sigma \in \operatorname{Ret} G$ and $H$ be a subgroup of $G$.

(1) If $\tau \in \operatorname{Ret} H$, then $\sigma$ extends $\tau$ if and only if $\operatorname{Ker} \tau \subseteq \operatorname{Ker} \sigma$.

(11) $H$ is a $\rho-\sigma$-subgroup of $G$ if and only if $\left\{g_{1}, \ldots, g_{n}\right\} \in \operatorname{Ker} \sigma$ and $h_{1}, \ldots, h_{n} \in H$ imply that $\left\{h_{1} g_{1}, \ldots, h_{n} g_{n}\right\} \sigma \in H$.

It was shown in [2, Theorem 4.3 (i)] that if $\sigma \in$ Ret $G$ and $H$ is a normal $\rho-\sigma$-subgroup of $G$, then the mapping $\sigma^{*}$ given by

$$
\left\{\mathrm{Hg}_{1}, \ldots, \mathrm{Hg}_{\mathrm{n}}\right\} \sigma *=\mathrm{H}\left(\left\{\mathrm{g}_{1}, \ldots, \mathrm{g}_{\mathrm{n}}\right\} \sigma\right)
$$

is a retraction of $G / H$ (called the retraction of $G / H$ induced by $\sigma$ ).

THEOREM 3.2: Let $G$ be a group, $H$ be a normal subgroup of $G$, and $\tau \in$ Ret $H$ such that for every $g \in G$ and every $A \in F(H),\left(g^{-1} A g\right) \tau=g^{-1}(A \tau) g$. Suppose further that $(\mathrm{G} / \mathrm{H}, \leq)$ is a linearly ordered group. Then there is a retraction $\sigma$ of $G$ that extends $\tau$. Moreover,

(i) $\mathrm{H}$ is a $\rho$ - $\sigma$-subgroup of $G, \sigma *$ is the retraction of $G / H$ induced by the linear ordering $\leq$ of $\mathrm{G} / \mathrm{H}$; and hence, $\mathrm{H}$ is a convex $\sigma$-subgroup of $\mathrm{G}$;

(ii) $\tau$ is convex if and only if $\sigma$ is convex;

(iii) $\tau$ is an $\ell$-retraction if and only if $\sigma$ is an e-retraction.

PROOF: Let $A=\left\{g_{1}, \ldots, g_{n}\right\} \in F(G)$ where

$$
\mathrm{Hg}_{1} \leq \ldots \leq \mathrm{Hg}_{\mathrm{p}-1}<\mathrm{Hg}_{\mathrm{p}}=\ldots=\mathrm{Hg}_{\mathrm{n}} \text {. }
$$


Then for $p \leq 1 \leq n, g_{1} g_{n}^{-1} \in H$. Let $h=\left\{g_{p} g_{n}^{-1}, \ldots, g_{n} g_{n}^{-1}\right\}_{\tau}$ and define $A \sigma=h g_{n}$. We must show that $\sigma$ is well-defined. Suppose that $d=\left\{g_{p} g_{p}^{-]}, \ldots, g_{n} g_{p}^{-1}\right\} \tau$. Since $g_{\mathrm{p}} \mathrm{g}_{\mathrm{n}}^{-1} \in \mathrm{H}$,

$$
d g_{p} g_{n}^{-1}=\left(\left\{g_{p} g_{p}^{-1}, \ldots, g_{n} g_{p}^{-1}\right\} \tau\right) g_{p} g_{n}^{-1}=\left\{g_{p} g_{n}^{-1}, \ldots, g_{n} g_{n}^{-1}\right\} \tau=h .
$$

Thus $\mathrm{dg}_{\mathrm{p}}=\mathrm{hg} \mathrm{g}_{\mathrm{n}}$ and it follows that $\sigma$ is a function. clearly, $\{\mathrm{g}\} \sigma=\mathrm{g}$ for every $g \in G$. Let $A$ be as above, $B=\left\{x_{1}, \ldots, x_{m}\right\} \in F(G)$, where $\mathrm{Hx}_{1} \leq \ldots \leq \mathrm{Hx} \mathrm{q}-1<\mathrm{Hx}_{\mathrm{q}}=\ldots=\mathrm{Hx} \mathrm{m}_{\mathrm{m}}$, and $\mathrm{d}=\left\{\mathrm{x}_{\mathrm{q}} \mathrm{x}_{\mathrm{m}}^{-1}, \ldots, \mathrm{x}_{\mathrm{m}} \mathrm{x}_{\mathrm{m}}^{-1}\right\} \tau$. Then $\mathrm{B} \sigma=\mathrm{dx} \mathrm{m}_{\mathrm{m}}$, and if

$$
\left\{g_{p} x_{q} x_{m}^{-1} g_{n}^{-1}, \ldots, g_{n} x_{m} x_{m}^{-1} g_{n}^{-1}\right\} \tau=c, \quad(A B) \sigma=c g_{n} x_{m}
$$

Now,

$$
\begin{aligned}
g_{n}^{-1} c g_{n} & =\left\{g_{n}^{-1} g_{p} x_{q} x_{m}^{-1}, \ldots, g_{n}^{-1} g_{n} x_{m} x_{m}^{-1}\right\} \tau \\
& =\left\{g_{n}^{-1} g_{p}, \ldots, g_{n}^{-1} g_{n}\right\} \tau\left\{x_{q} x_{m}^{-1}, \ldots, x_{m} x_{m}^{-1}\right\} \tau \\
& =g_{n}^{-1} g_{n} d .
\end{aligned}
$$

Therefore, $c g_{n}=h g_{n} d$ and so $(A B) \sigma=c g_{n} x_{m}=h g_{n} d x_{m}=(A \sigma)(B \sigma)$. Hence $\sigma \in \operatorname{Ret} \mathrm{G}$.

If $\left\{h_{1}, \ldots, h_{n}\right\} \in \operatorname{Ker} \tau$, then $h_{n}^{-1}=\left\{h_{1} h_{n}^{-1}, \ldots, h_{n} h_{n}^{-1}\right\} \tau$ and so $\left\{h_{1}, \ldots, h_{n}\right\} \sigma$ $=h_{n} h_{n}^{-1}=\imath$. Thus Ker $\tau \subseteq \operatorname{Ker} \sigma$ and, by Theorem 3.1, $\sigma$ extends $\tau$.

The verification of (i) is immediate from Theorem 3.1 and [2, Theorem 4.3 (i1)], the verification of (ii) is straightforward, and (iii) is well-known from the theory of lattice-ordered groups.

COROLLARY 3.3: If $G$ is a torsion-free abelian group, $\mathrm{H}$ is a proper pure subgroup of $G$, and $\tau \in \operatorname{Ret} H$, then $\tau$ can be extended to a retraction of $G$ in at least two ways.

PROOF: If $H$ is a pure subgroup of $G$, then $G / H$ is torsion-free. Each torsion-free abelian group can be linearly ordered [5, p. 0.4]. Since $H$ is a proper subgroup of $G, G / H$ admits at least two distinct linear orderings. Hence $\tau$ can be extended in at least two ways to a retraction of $G$. 


\section{DIVISIBLE EXTENTIONS.}

The main result of this section is that each retraction of a torsion-free abelian group can be uniquely extended to a retraction of its divisible closure. Similar results for partially ordered groups may be found in [9], [6], or [5, Chap. 4].

If $G$ is a lattice-ordered group, $g_{1}, \ldots, g_{m} \in G$ such that $g_{i} g_{j}=g_{j} g_{i}$ for all $i$ and $j$, and $n \in N$, then $\left(g_{1} \vee \ldots v g_{m}\right)^{n}=g_{1}^{n} v \ldots v g_{m}^{n}[5, p .0 .17]$. In Theorem 4.3 we show that this identity generalizes to retractable groups. To this end, we state two lemmas. (A proof of Lemma 4.1 can be given by induction and Lemma 4.1 can be used to prove Lemma 4.2.)

LEMMA 4.1: If $G$ is a group, $A=\left\{g_{1}, \ldots, g_{m}\right\} \in F(G)$ such that $g_{i} g_{j}=$ $g_{i} g_{i}$ for all $i$ and $j$, and $n \in N$, then

$$
A^{n}=\left\{g_{1}{ }^{k_{1}} \ldots g_{m} k_{m} \mid \text { if } 1 \leq r \leq m \text {, then } k_{r} \geq 0 \text { and } \sum_{r=1}^{m} k_{r}=n\right\} \text {. }
$$

LEMMA 4.2: If $G$ is a group, $A=\left\{g_{1}, \ldots, g_{m}\right\} \in F(G)$ such that $g_{i} g_{j}=g_{j} g_{i}$ for all $i$ and $j$, and $n \in N$, then for each $p \in N$ such that $p \geq(m-1)(n-1)$,

$$
A^{p}\left\{g_{1}^{n}, \ldots, g_{m}^{n}\right\}=A^{p+n} .
$$

THEOREM 4.3: If $\sigma \in \operatorname{Ret} G, A=\left\{g_{1}, \ldots, g_{m}\right\} \in F(G)$ such that $g_{i} g_{j}=g_{j} g_{i}$ for all $i$ and $j$, and $n \in N$, then $\left\{g_{1}^{n}, \ldots, g_{m}^{n}\right\} \sigma=(A \sigma)^{n}$.

PROOF: Since $\sigma$ is a homomorphism, $(A \sigma)^{n(m+1)}=\left(A^{n(m+1)}\right) \sigma$, and by Lemma 4.2, $A^{n(m+1)}=A_{A}^{n m}\left\{g_{1}^{n}, \ldots, g_{m}^{n}\right\}$. Therefore, $(A \sigma)^{n(m+1)}=\left(A^{n m^{n}}\left\{g_{1}^{n}, \ldots, g_{m}^{n}\right\}\right) \sigma=$ $(A \sigma)^{n m}\left(\left\{g_{1}^{n}, \ldots, g_{m}^{n}\right\}\right) \sigma$. Hence, $\left\{g_{1}^{n}, \ldots, g_{m}^{n}\right\} \sigma=(A \sigma)^{n}$.

We state the following three corollaries which will be used numerous times in the sequel.

COROLLARY 4.4: If $G$ is an abelian group and $\sigma \in$ Ret $G$, then $\sigma$ satisfies condition $(\delta)$.

COROLLARY 4.5: If $G$ is an abelian group, $n \in N, G^{(n)}=\left\{g^{n} \mid g \in G\right\}$, and $\sigma \in \operatorname{Ret} G$, then $G(n)$ is a $\sigma$-subgroup of $G$. Thus, the largest divisible 
subgroup of $G$ is a $\sigma$-subgroup.

COROLLARY 4.6: Let $G$ be an abelian group, $H$ a subgroup of $G$, and

$$
H_{*}=\left\{g \mid g \in G \text { and } g^{n} \in H \text { for some } n \in N\right\}
$$

be the pure closure of $H$ in $G$. If $\sigma \in \operatorname{Ret} G$ and $H$ is a $\sigma$-subgroup of $G$, then so is $\mathrm{H}_{*}$.

Let $G$ be a torsion-free abelian group and $D$ be a divisible closure of $G$. For $n \in N$, let

$$
D_{n}=\left\{d \mid d \in D \text { and } d^{n} \in G\right\}
$$

Then $D_{n}$ is a subgroup of $D, G=\underset{n \in N}{n} D_{n}, D=\underset{n \in N}{u} D_{n}, D_{m} \cap D_{n}=D_{g \cdot c \cdot d}(m, n)$, and $D_{m} D_{n}=D_{1 . c \cdot m .(m, n)}$. If $g \in G$, we write $g^{1 / n}$ for that unique element in $D$ whose $n$-th power is $g$. For $n \in N$, define $\theta_{n}$ from $G$ into $D$ by $g \theta_{n}=g{ }^{1 / n}$. Then $\theta_{n}$ is an isomorphism of $G$ onto $D_{n}$.

THEOREM 4.7: If $G$ is an abelian group, $D$ is a divisible closure of $G$, and $\sigma \in \operatorname{Ret} G$, then $\sigma$ can be uniquely extended to a retraction $\tau$ of $D$. Moreover,

(i) for each $\mathrm{n} \in \mathrm{N}, \mathrm{D}_{\mathrm{n}}$ is a $\tau$-subgroup of $\mathrm{D}$;

(ii) $\operatorname{Ker} \tau=\left\{\left\{g_{1}^{1 / n}, \ldots, g_{m}^{1 / n}\right\} \mid n \in N\right.$ and $\left.\left\{g_{1}, \ldots, g_{m}\right\} \in \operatorname{Ker} \sigma\right\}$ and Ker $\sigma=F(G) \cap \operatorname{Ker} \tau$;

(iii) Ker $\sigma$ is a convex subset of $F(G)$ if and only if $\operatorname{Ker} \tau$ is a convex subset of $F(D)$;

(iv) $\operatorname{Ker} \sigma$ is a convex subsemilattice of $F(G)$ if and only if $\operatorname{Ker} \tau$ is a convex subsemilattice of $F(D)$; hence $\sigma$ is an $\ell$-retraction of $F$ if and only if $\tau$ is an l-retraction of $D$.

PROOF: For each $n \in N$, let $\sigma_{n}=A_{n}^{-1} \sigma \theta_{n}$, where $\theta_{n}$ is given above. That

$$
\begin{aligned}
& \text { is, for } A=\left\{g_{1}, \ldots, g_{m}\right\} \in F\left(D_{n}\right), \\
& \qquad A \sigma_{n}=\left(\left\{g_{1} \theta_{n}^{-1}, \ldots, g_{m} \theta_{n}^{-1}\right\} \sigma\right) \theta_{n} .
\end{aligned}
$$

Then $\sigma_{n} \in$ Ret $D_{n}$. Let $\tau=\underset{n \in N}{U} \sigma_{n}$. The verification that $\tau$ is the unique 
extension of $\sigma$ to a retraction of $D$, and the remainder of the theorem is routine.

COROLLARY 4.8: Let $G$ be a torsion-free abelian group and $H$ be a subgroup of $G$. If there exists $\tau \in \operatorname{Ret} H$ such that $\tau$ has a unique extension to $G$, then $G / H$ is torsion (or equivalently, $G$ is contained in a divisible closure of $\mathrm{H}$ ).

PROOF: Let $\sigma$ be the unique extension of $\tau$ to a retraction of $G$. If $\mathrm{H}_{\star}$ denotes the pure closure of $\mathrm{H}$ in $\mathrm{G}$, then by Corollary $4.6, \mathrm{H}_{\star}$ is a $\sigma-$ subgroup of G. Hence, $\tau$ extends uniquely to a retraction of $H_{\star} \cdot$ Thus, by Corollary 3.3, $\mathrm{H}_{\star}=\mathrm{G}$.

If $G$ is a torsion-free abelian group, $D$ is a divisible closure of $G$, $\sigma \in \operatorname{Ret} G$, and $\tau$ is the unique extension of $\sigma$ to a retraction of $D$, then it is easy to show that the collection of $\rho$ - $\sigma$-subgroups of $G$ is lattice isomorphic to the $\rho$ - $\tau$-subgroups of $D$.

\section{EXTENDING RETRACTIONS.}

In this section we prove the main result (Theorem 5.7) of this paper, namely, if $G$ is a torsion-free ableian group and $H$ is a subgroup of $G$, then we give a necessary and sufficient condition that a retraction of $H$ can be extended to a retraction of $\mathrm{G}$.

THEOREM 5.1: Let $G$ be an abelian group, $D$ a divisible closure of $G$, $K$ be a subgroup of $D$ that contains $G$, and $\sigma \in$ Ret $G$. Then $\sigma$ can be extended to a retraction of $K$ if and only if for each $n \in N, K^{(n)} \cap G$ is a $\sigma$-subgroup of G. Moreover, if $\sigma$ extends to a retraction of $K$, then it is unique.

PROOF: Let $\tau$ be the unique extension of $\sigma$ to a retraction of $D$. If $\sigma$ can be extended to a retraction $\nu$ of $K$, then $\tau$ is the unique extension of $\nu$ to a retraction of D. If $n \in N$, then by Corollary 4.5, $K^{(n)}$ is a -subgroup of $K$ and hence, a $\tau$-subgroup of $D$. Therefore, $K^{(n)} \cap G$ is a 
$\tau$-subgroup of D. Since $\tau \mid F(G)=\sigma$, it follows that $K^{(n)} \cap G$ is a $\sigma$-subgroup of $G$.

Conversely, suppose that $K^{(n)} \cap G$ is a $\sigma$-subgroup of $G$ for each $n \in N$. Let $\left\{h_{1}, \ldots, h_{m}\right\} \in F(K)$ and $\left\{h_{1}, \ldots, h_{m}\right\} \tau=a$. Then there exists $n \in N$ such that $\left\{h_{1}^{n}, \ldots, h_{m}^{n}\right\} \in F(G)$. Since $\tau$ satisfies $(\delta)$,

$$
a^{n}=\left\{h_{1}^{n}, \ldots, h_{m}^{n}\right\} \tau=\left\{h_{1}^{n}, \ldots, h_{m}^{n}\right\} \sigma .
$$

Now, $K^{(n)} \cap G$ is a $\sigma$-subgroup of $G$ and $\left\{h_{1}^{n}, \ldots, h_{m}^{n}\right\} \in F\left(K^{(n)} \cap G\right)$. Hence, $a^{n}=\left\{h_{1}^{n}, \ldots, h_{m}^{n}\right\} \sigma \in K^{(n)} \cap G$. Since $D$ is torsion-free, a $\in K$. Therefore, $K$ is a $\tau$-subgroup of $D$ and hence, $\sigma$ can be extended to a retraction of $K$.

The uniqueness is immediate from Theorem 4.7.

From the proof of Theorem 5.1, we have

COROLLARY 5.2: Let $G$ be an abelian group, $D$ be a divisible closure of $G$, $K$ be a subgroup of $D$ that contains $G$, and $\sigma \in$ Ret $G$. If there exists $n \in N$ such that $K^{(n)} \subseteq G$ and $K^{(n)}$ is a $\sigma$-subgroup of $G$, then $\sigma$ has a unique extension to a retraction of $\mathrm{K}$.

In the following considerations (up to Corollary 5.6), for obvious reasons, we use additive notation for the binary operations on groups. Let $H$ be a subgroup of $Q$ and define $H^{\perp}=\{r \mid r \in Q$ and $r h \in H$ for every $h \in H\}$. It is easily verified that $\mathrm{H}^{\perp}$ is a subring of $Q$ containing $Z$. Moreover, it follows from the theory of abelian groups of rank 1 that if $K$ is a subgroup of $Q$ containing $\mathrm{H}$ and $\mathrm{H} \neq\{0\}$, then $\mathrm{H}^{\perp}$ is a subring of $\mathrm{K}^{\perp}$.

In [2, Example 5.7] all of the retractions of $Q$ were exhibited, namely, Ret $Q=\left\{\sigma_{r} \mid r \in Q\right\}$, where $\sigma_{r}$ is defined by $A \sigma_{r}=(r+1) \max A-r \min A$, for al1 $A \in F(Q)$.

COROLLARY 5.3: If $H$ is a nonzero subgroup of $Q$, then Ret $H=\left\{\sigma_{r} \mid r \in H^{\perp}\right\}$. COROLLARY 5.4: If $H$ and $K$ are subgroups of $Q$ such that $H \subseteq K, H \neq\{0\}$, and $\sigma \in$ Ret $\mathrm{H}$, then $\sigma$ can be uniquely extended to a retraction of $\mathrm{K}$. The preceding corollary is not true for abelian groups of rank 2, as we 
illustrate with the following example.

EXAMPLE 5.5: Let $K=Z \times Z$ and let $\sigma$ be the -retraction of $K$ induced by the cardinal ordering of $K$. Let $H=K+\left\langle\left(\frac{1}{2},-\frac{1}{2}\right)\right\rangle$, where $\left\langle\left(\frac{1}{2},-\frac{1}{2}\right)\right\rangle$ denotes the cyclic subgroup of $Q \times Q$ generated by $\left(\frac{1}{2},-\frac{1}{2}\right)$. Then $2 \mathrm{H}=2 \mathrm{~K}+\langle(1,-1)\rangle$ and $(1,-1) \in 2 \mathrm{H} \cap \mathrm{K}$. Now $\{(0,0),(1,-1)\} \sigma=(0,0) \vee(1,-1)=(1,0) \in \mathrm{K}$. However, $(1,0) \notin 2 \mathrm{H}$. Thus, $2 \mathrm{H} \cap \mathrm{K}$ is not a $\sigma$-subgroup of $\mathrm{K}$ and so by Theorem 5.1, $\sigma$ can not be extended to a retraction of $\mathrm{H}$.

The next corollary is an immediate consequence of Corollary 5.2.

COROLLARY 5.6: If $G$ is a torsion-free abelian group, $n \in N$, and $\sigma \in \operatorname{Ret} G^{(n)}$, then $\sigma$ has a unique extension to a retraction on $G$.

We next turn our attention to the problem of extending a retraction of a subgroup $\mathrm{H}$ of a torison-free abelian group $\mathrm{G}$ to a retraction of $\mathrm{G}$.

THEOREM 5.7: Let $G$ be a torsion-free abelian group, $H$ a subgroup of $G$, and $\tau \in$ Ret $\mathrm{H}$. Then a necessary and sufficient condition that $\tau$ can be extended to a retraction of $G$ is that $G(n) \cap H$ is a $\tau$-subgroup of $H$ for each $\mathrm{n} \in \mathrm{N}$.

PROOF: Suppose that $G^{(n)} \cap H$ is a $\tau$-subgroup of $H$ for each $n \in N$. We first show that there is a pure subgroup $M$ of $G$ containing $H$ such that $\tau$ can be extended to a retraction of $M$. Let $M=\{(M, \nu \mid M$ is a subgroup of $G$ that contains $H, \nu \in \operatorname{Ret} M, \nu \mid F(H)=\tau$, and $G^{(n)} \cap M$ is a $\nu$-subgroup of $M$ for each $n \in N\}$. Then $(H, \tau) \in M$ and for $\left(M_{1}, \nu_{1}\right),\left(M_{2}, \nu_{2}\right) \in M$, define $\left(M_{1}, \nu_{1}\right) \leq\left(M_{2}, \nu_{2}\right)$ if and only if $M_{1} \subseteq M_{2}$ and $\nu_{2} \mid F\left(M_{1}\right)=\nu_{1}$. By Zorn's Lemma, there exists a maximal element $(M, \nu)$ of $M$. We show that $M$ is a pure subgroup of $G$. Let $D$ be a divisible closure of $G$ and let $C$ be the divisible closure of $M$ in $D$. Then, by Theorem 4.7, $\nu$ can be extended to a retraction $\mu$ of $\mathrm{C}$. Let $\mathrm{X}=\mu \mid \mathrm{F}(\mathrm{G} \cap \mathrm{C})$. Then, we assert that $(G \cap \mathrm{C}, \mathrm{X}) \in M$. If $\mathrm{n} \in \mathrm{N}$ and $\left\{c_{1}, \ldots, c_{m}\right\} \in F(G(n) \cap C)$, then $G^{(n)} \cap(G \cap C)=G^{(n)} \cap C$, and there exists $p \in N$ such that $\left\{c_{1}^{p}, \ldots, c_{m}^{p}\right\} \subseteq G^{(n p)} \cap M$. Since $\mu$ satsifies $(\delta)$ and 
$(M, \nu) \in M,\left(\left\{c_{1}, \ldots, c_{m}\right\}_{\mu}\right)^{p}=\left\{c_{1}^{p}, \ldots, c_{m}^{p}\right\}_{\mu}=\left\{c_{1}^{p}, \ldots, c_{m}^{p}\right\}_{X}=\left\{c_{1}^{p}, \ldots, c_{m}^{p}\right\}_{\nu \epsilon}$ $G^{(n p)} \cap M$. Hence, $\left\{c_{1}, \ldots, c_{m}\right\} X=\left\{c_{1}, \ldots, c_{m}\right\} \mu \in G{ }^{(n)} \cap C$. In particular, when $\mathbf{n}=1$, we have shown that,$\chi \in \operatorname{Ret}(G \cap C)$, and for $\mathbf{n}$ arbitrary $(\mathbf{n}>0)$, $G^{(n)} \cap C$ is a $X$-subgroup of $G \cap C$. Therefore, $(G \cap C, X) \in M$ and by the maximality of $(M, \nu), G \cap C=M$. Consequently, $M$ is pure in $G$. By Corollary 3.3, $V$ can be extended to a retraction of $G$ and hence, $\tau$ can be extended to a retraction of $\mathrm{G}$.

The converse is immediate by Corollary 4.5.

By Corollary 5.3, Ret $z=\left\{\sigma_{r} \mid r \in Z^{\perp}\right\}=\left\{\sigma_{r} \mid r \in Z\right\}$. Thus, for each subgroup $H$ of $Z$ and each $r \in \operatorname{Ret} Z, H$ is a $\sigma$-subgroup of $Z$. It follows that if $G$ is a torsion-free abelian group and $g \in G$, then each retraction of the cyclic subgroup of $G$ generated by $g$ can be extended to a retraction of G. (Hence, each torsion-free abelian group has at least a countably infinite number of retractions.) Combining Corollary 5.4 and Theorem 5.7, we have a stronger result than this.

COROLLARY 5.8: Let $G$ be a torsion-free abelian group and $H$ be a locally cyclic subgroup of $G$. Then every retraction of $H$ can be extended to a retraction of $G$.

\section{REFERENCES}

1. G. Birkhoff, Lattice Theory, 3rd. ed., Amer. Math. Soc. Colloq. Publ. vol. 25, Providence, 1967.

2. R. D. Byrd, J. T. Lloyd, R. A. Mena, and J. R. Teller, Retractable groups, Acta. Math. Acad. Sci. Hungar.29(1977), 219-233.

3. R. D. Byrd, J. T. Lloyd, R. A. Mena, On the retractability of some one-relator groups, Pacific J. Math. 72(1977), 351-359.

4. R. D. Byrd, J. T. Lloyd, F. D. Pedersen, and J. W. Stepp, Automorphisms of the semigroup of finite complexes of a torsion-free abelian group, submitted.

5. P. Conrad, Lattice-ordered groups, Lecture notes, Tulane University, New Orleans, 1970. 
6. P. Conrad, J. Harvey, and C. Holland, The Hahn embedding theorem for abelian lattice-ordered groups, Trans. Amer. Math. Soc. 108 (1963), 143-169.

7. L. Fuchs, Partially Ordered Algebraic Systems, Pergamon Press, New York, 1963.

8. L. Fuchs, Infinite Abelian Groups, Vo1. I, Academic Press, New York, 1970.

9. J. Jakubik, Representation and extensions of $\ell$-groups (Russian, German summary), Czech. Math. J. 13 (1967), 267-283.

10. F. Levi, Ordered groups, Proc. Indian Acad. Sc1., Sect. A, 16(1942), 256-263.

11. J. Rotman, The Theory of Groups, 2nd ed., Allyn and Bacon, Boston, 1973. 


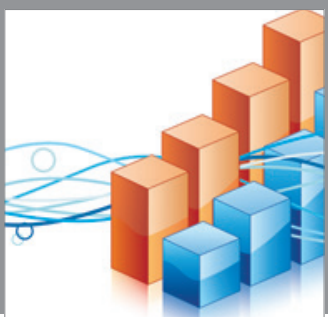

Advances in

Operations Research

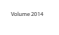

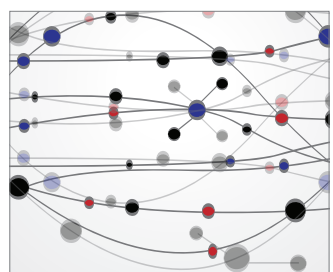

\section{The Scientific} World Journal
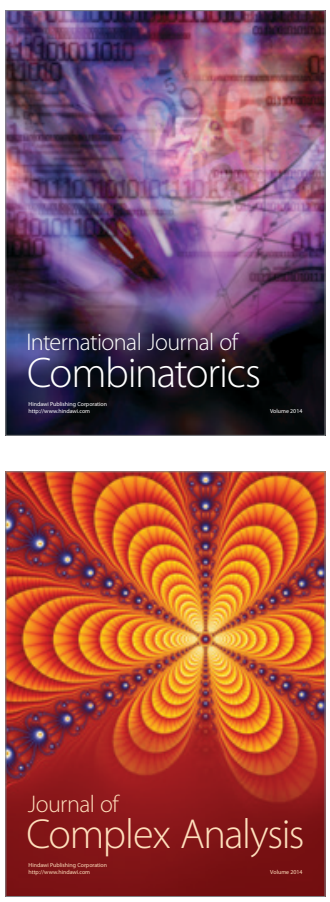

International Journal of

Mathematics and

Mathematical

Sciences
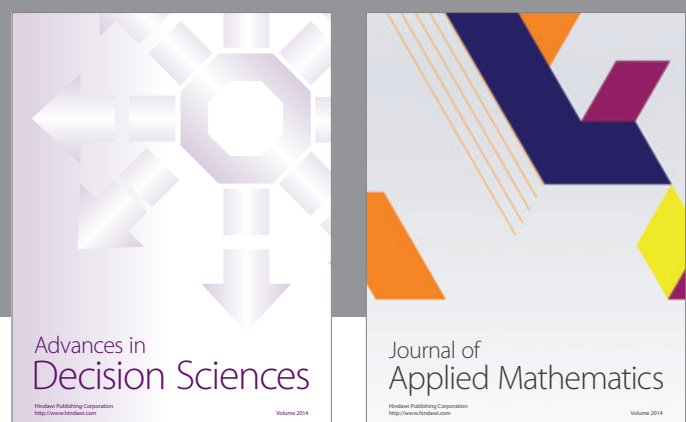

Journal of

Applied Mathematics
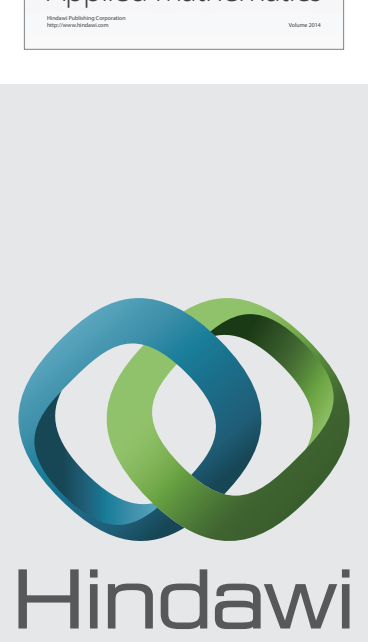

Submit your manuscripts at http://www.hindawi.com
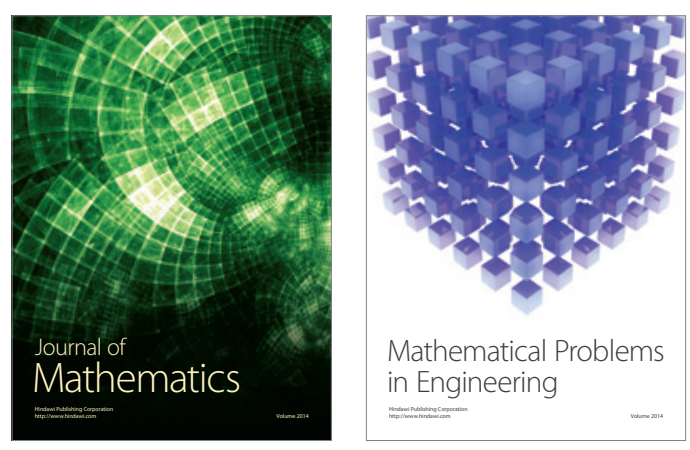

Mathematical Problems in Engineering
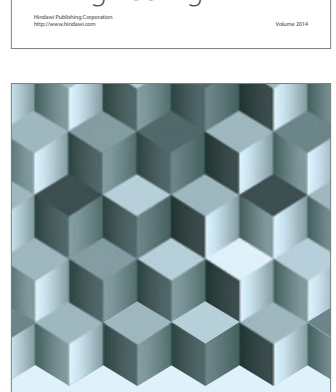

Journal of

Function Spaces
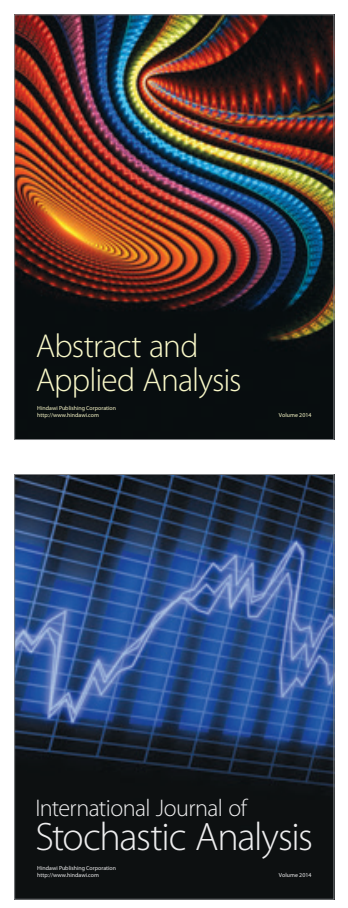

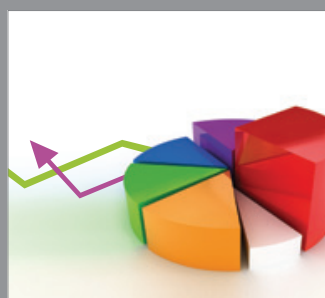

ournal of

Probability and Statistics

Promensencen
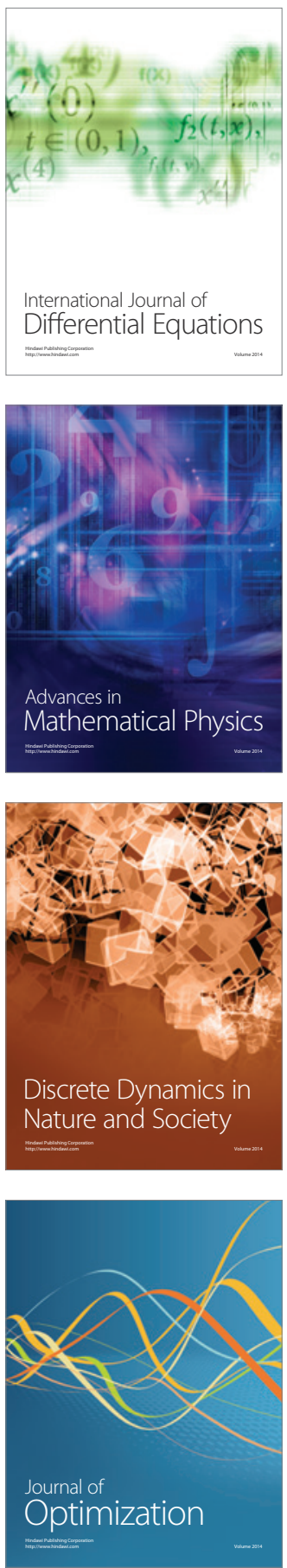